“드 2013 IEEE. Personal use of this material is permitted. Permission from IEEE must be obtained for all other uses, in any current or future media, including reprinting/republishing this material for advertising or promotional purposes, creating new collective works, for resale or redistribution to servers or lists, or reuse of any copyrighted component of this work in other works." 


\title{
Unsupervised segmentation of heel-strike IMU data using rapid cluster estimation of wavelet features
}

\author{
Mitchell Yuwono, Steven W. Su, Bruce D. Moulton, and Hung T. Nguyen
}

\begin{abstract}
When undertaking gait-analysis, one of the most important factors to consider is heel-strike (HS). Signals from a waist worn Inertial Measurement Unit (IMU) provides sufficient accelerometric and gyroscopic information for estimating gait parameter and identifying HS events. In this paper we propose a novel adaptive, unsupervised, and parameter-free identification method for detection of HS events during gait episodes. Our proposed method allows the device to learn and adapt to the profile of the user without the need of supervision. The algorithm is completely parameter-free and requires no prior fine tuning. Autocorrelation features (ACF) of both anteroposterior acceleration $\left(a_{A P}\right)$ and medio-lateral acceleration $\left(a_{M L}\right)$ are used to determine cadence episodes. The Discrete Wavelet Transform (DWT) features of signal peaks during cadence are extracted and clustered using Swarm Rapid Centroid Estimation (Swarm RCE). Left HS (LHS), Right HS (RHS), and movement artifacts are clustered based on intra-cluster correlation. Initial pilot testing of the system on 8 subjects show promising results up to $84.3 \% \pm 9.2 \%$ and $86.7 \% \pm 6.9 \%$ average accuracy with $86.8 \% \pm 9.2 \%$ and $88.9 \% \pm 7.1 \%$ average precision for the segmentation of LHS and RHS respectively.
\end{abstract}

\section{INTRODUCTION}

The analysis of gait parameters is beneficial for assessing treatment effectiveness, quality of mobility and general health [1], [2]. Information about gait parameters provides important diagnostics for balance, functional ability, risk of falls [1].

The current methods for assessing gait parameters are mostly laboratory-based. They are expensive and not practical for application in daily life [3]. Force platforms, as the gold standard, can be used to precisely record the ground reaction forces exerted by the feet during the gait cycle [4]. Other popular methods use lower-limb sensors [5], pressure insoles [6], or stereo-photogrammetric cameras [7].

A waist-worn Inertial Measurement Unit (IMU) is a lowcost solution for extracting gait parameters [1]-[3], [8]. An IMU consists of an accelerometer and a gyroscope. Using a single waist-worn accelerometer, Moe-Nilssen and Tura estimate gait regularity from the autocorrelation function (ACF) pattern of the mediolateral (ML) and anteroposterior (AP) acceleration [1], [3]. Using similar setup, Bugané extracts the gait parameters by identifying important gait events including heel-strikes (HS) and toe-offs (TO) based on peak detection and thresholding of the AP and ML acceleration [8]. Similarly, Köse use the stationary wavelet transform and peak detection to extract HS \& TO events using a single IMU worn on the lateral side of the pelvis to finally calculate bilateral step length estimate [2].

\footnotetext{
M. Yuwono, S.W. Su, B. D. Moulton, and H. T. Nguyen are with the Faculty of Engineering and Information Technology, University of Technology, Sydney, Ultimo, 2007, NSW, Australia. email: mitchellyuwonolgmail.com
}

The above methods tend to perform poorly in real-world situations where the data is noisy, where gait patterns vary in real-time, and where there is a degree of drift in the placement of the sensors. To reliably identify HS using an IMU, the system should be able to distinguish movement artifacts from HS acceleration patterns. In this paper we propose a parameterfree HS and stride pattern clustering. The proposed protocol allows the system to adapt to the user gait pattern over time.

Section II gives an overview of the hardware and software. Section III describes the feature extraction methods. Section IV explains the feature clustering results. Section V describes the experimental settings, results and analysis. Finally Section VI provides the conclusion and future directions.

\section{OVERVIEW}

\section{A. Hardware and Software}

We use the Shimmer MEMS kinematic module with a Wireless 9DoF Kinematic daughterboard IMU. The base package of Shimmer contains a Freescale MMA7361 triaxial accelerometer. The daughterboard provides a Honeywell HMC5843 magnetometer, and an InvenSense500 gyroscope [9]. The sensor is attached to a belt and positioned on the right side of the ML axis, similarly to the setup of Köse [2]. The device has been calibrated such that the positive $\mathrm{x}$-axis points downwards towards the gravity vector, positive $y$-axis points forward towards AP vector, and positive z-axis points sideways towards ML vector. The IMU is sampled at $50 \mathrm{~Hz}$. Prototyping is done using Matlab.

\section{B. Algorithm}

A gait sequence is detected using the frequency profile of both Vertical acceleration $\left(a_{V}\right)$ and ML acceleration $\left(a_{M L}\right)$ [1]. A peak detection algorithm is applied to the cadence signal, particularly the $a_{V}$ segment, to extract HS, TO, and movement artifacts. On each peak location, gyroscopic and accelerometric wavelet features are calculated.

The HS features are clustered to 3 classes which are LHS, RHS, and outliers including TO events and movement artifacts. LHS and RHS patterns can be recognized based on the clustered wavelet features. Consecutive HS of the same foot with similar time difference with $a_{M L}$ ACF peak is detected as a stride [1]. The user's bilateral stride profile can then be analyzed from the recognized signals.

\section{FEATURE EXTRACTION}

The signals measured from a waist-worn IMU provides rich information that can be used to estimate spatio-temporal gait parameters, gait events, and gait phases [1]-[3], [8], [10]. This 
section provide our proposed feature extraction method for our experimental configuration.

\section{A. Cadence and Stride Rate Estimation using ACF}

Moe-Nilssen proposes that gait parameters can be extracted by examining the ACF of vertical acceleration $a_{V}$ and ML acceleration $a_{M L}$ [1]. Cadence estimate is found by measuring the fundamental frequency of $a_{V}$ ACF. Stride rate is found by performing similar calculations on $a_{M L}$ signal.

We have previously used spectrogram analysis and image processing techniques to detect gait episodes with promising result [11]. We identify cadence as a tonal frequency over prolonged time period. Cadence frequency ranges between 0.6 to $2.5 \mathrm{~Hz}$ (steps per seconds) or 36 to 150 steps per minute. In this work we simplify the prior algorithm to estimate cadence using informations from $a_{A P}$ and $a_{M L}$ signals.

A cadence can be estimated as follows: We take subsegments with interval of $\Delta t$ from $a_{A P}$ and $a_{M L}$ signals. Both signals are filtered using a fourth order Butterworth filter with cutoff frequencies of 0.5 and $3 \mathrm{~Hz}$. Fundamental frequencies $f_{0 A P}$ and $f_{0 M L}$ are approximated by calculating the spectral centroids from the resulting power spectra. The AP timefrequency continuity $f_{0 A P}\left(\Delta t_{0 A P}\right)$ represents cadence, while the ML time-frequency continuity $f_{0 M L}\left(\Delta t_{0 M L}\right)$ represents stride rate. A cadence estimate is valid at time $\Delta \tau: \Delta \tau \in$ $\Delta t_{0 A P} \cap \Delta t_{0 M L}$ when the condition $f_{0 A P}(\Delta \tau) / f_{0 M L}(\Delta \tau) \approx 2 \pm$ 0.5 is satisfied. The pseudocode is shown in Algorithm 1.

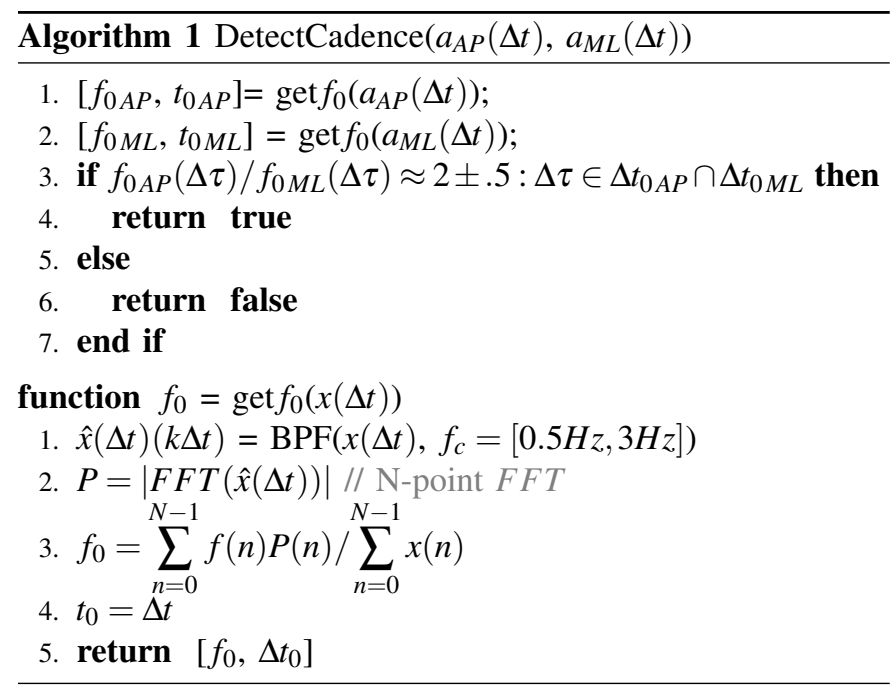

\section{B. HS Wavelet Features Extraction}

The discrete wavelet transform (DWT) detects frequency localizations at specific times in a signal. A large value at a time-frequency localization indicates high similarity between the mother wavelet and the signal at the specified instant. This quality is especially useful for extracting instantaneous pattern and frequency changes such as ones imposed by HS and TO events [4].

HS events produces signals that are morphologically similar to the Debauchies 4-tap wavelet (db4). This can be seen by observing the peaks and valleys in the sensory signals around HS events. HS frequencies are localized around $6-8 \mathrm{~Hz}$ frequency slot. For $f_{s}=50 \mathrm{~Hz}$, DWT at the $2^{\text {nd }}$ level of decomposition effectively focuses on frequency localization around $6.25-12.5 \mathrm{~Hz}$. We propose that using our configuration, $2^{\text {nd }}$ level DWT is appropriate for detecting HS events.

The wavelet features that we use are: the $2^{\text {nd }}$ level detail coefficients (d2) of $a_{A P}, a_{M L}, a_{V}, \omega_{S}$, and $\omega_{T}$; and the $2^{\text {nd }}$ level approximation coefficients (a2) of $\omega_{C}$. Each coefficient dimensionality is 11. The total dimension of a HS data is 66.

A particular subject's LHS and RHS pattern and their wavelet features are presented in Figure 1. The subject was walking with cadence of $111.9 \pm 7$ steps/minute.
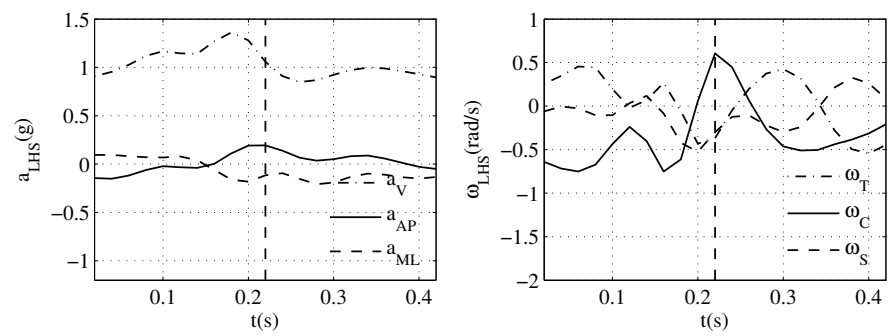

(a) LHS raw signals
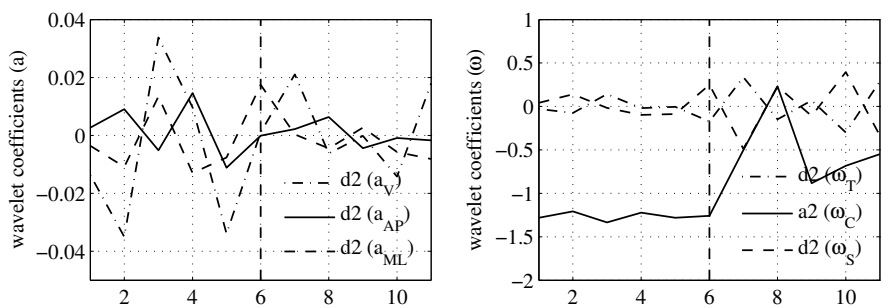

(b) LHS DWT features
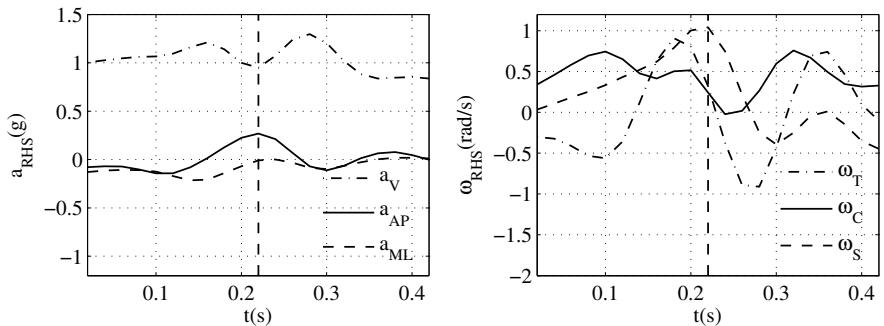

(c) RHS raw signals
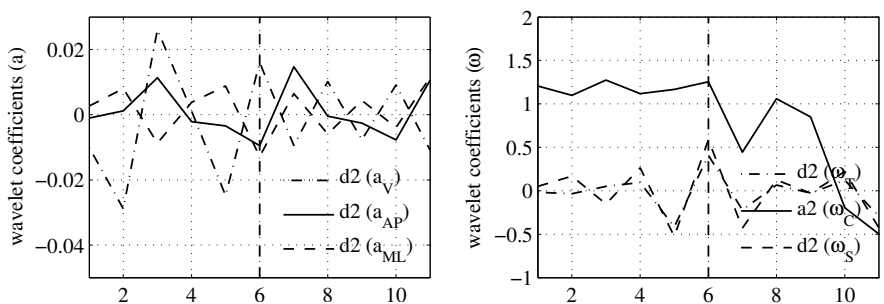

(d) RHS DWT features

Figure 1: LHS and RHS pattern of subject 7 averaged over 273 RHS and 276 LHS events. Subject was walking with cadence of $111.9 \pm 7$ steps/minute. 


\section{Feature Clustering}

The general template HS signals have been proposed in literatures [2], [8]. However, HS pattern of individuals change over time and are affected by clothes, footwear, walking surface, cadence, and emotional condition [12]. We propose that a data-driven approach using data clustering is appropriate for this particular task. The intention is to design a system that is able to adapt to pattern changes.

We have recently proposed a lightweight clustering algorithm using swarm-intelligence we term the Rapid Centroid Estimation (RCE) [13], [14]. We encourage interested readers to refer to [13] and [14] for further information.

\section{A. HS Clustering}

Using RCE, we cluster the wavelet features of the peaks to segment RHS, LHS and TO events. The cluster optimization function is the sum of intra-cluster correlation distance. Correlation distance is selected in order to preserve intra-cluster pattern similarity. A particular clustered data distribution is shown in Figure 2. We observed that choosing three clusters provides a representative model based on the visualization of the feature distribution.

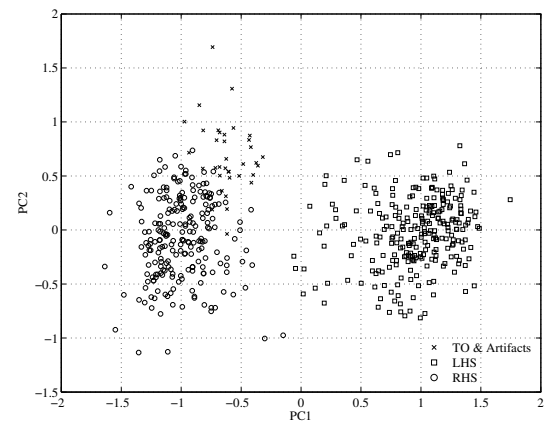

Figure 2: RCE clustering results of wavelet features.

\section{B. Stride Profile}

A stride is indicated by a consecutive HS of the same foot. A valid stride satisfies the following criteria:

1) The consecutive HS of the same foot have similar timing to the first ACF peak of $a_{M L}$ and the second peak of $a_{A P}$ and $a_{V}$.

2) The HS of the opposite foot have similar timing to the first peak of $a_{A P}$ and $a_{V}$.

The stride profile of Subject 7 is presented in Figure 3a. The corresponding ACF profile is presented in Figure $3 b$.

\section{ExPerimental Results and Discussion}

We tested this method with 8 healthy subjects (5 male, 3 female) aged between 20 and 67 years old). A Shimmer 9DoF IMU was attached to the subject's waist at the right side of the ML axis. Each subject was told to walk for five minutes at a personally selected pace. The number of strides are counted. The experimental data collection is done in a house environment to simulate daily living condition.
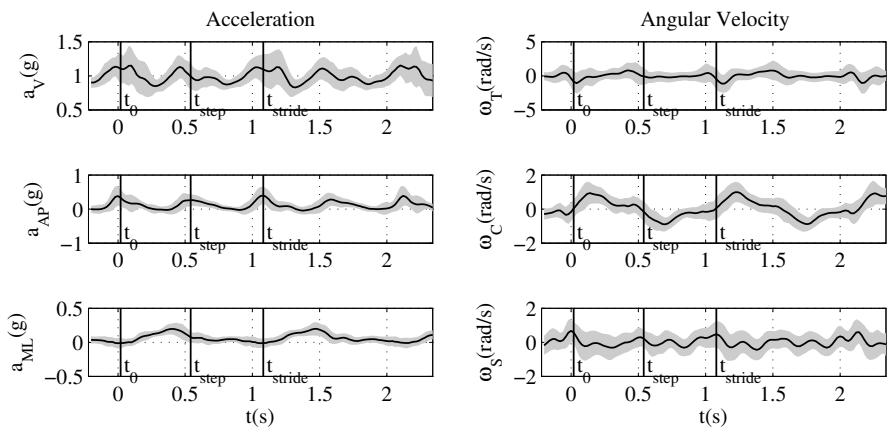

(a) Right leg stride accelerometric and gyroscopic signal pattern.
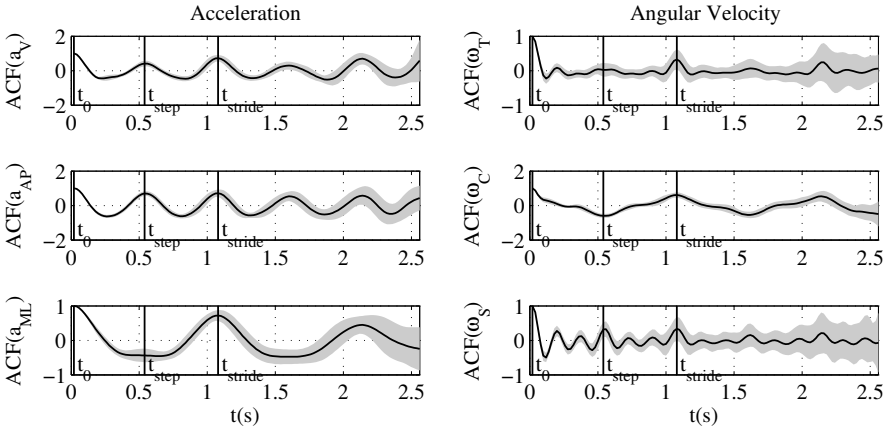

(b) The corresponding ACF of Signals in Figure 3a

Figure 3: Right leg stride accelerometric and gyroscopic signal pattern of Subject 7 averaged over 238 successive strides.

The gait parameters, including cadence and step symmetry, are calculated using Moe-Nilssen's method [1]. Accuracy is calculated by dividing HS positive detection rate by total number of steps. Precision is calculated by dividing HS true positive detection by the total number of true and false positives. The HS profile for each subject is obtained by averaging correct detections. The general HS profile for all subject is obtained by averaging each subject's HS profiles. Table I shows the experimental results.

Table I shows that our proposed method has on average $84.3 \% \pm 9.2 \%$ and $86.7 \% \pm 6.9 \%$ accuracy with $86.8 \% \pm 9.2 \%$ and $88.9 \% \pm 7.1 \%$ precision on LHS and RHS segmentation. The LHS and RHS profile of each user are also unique to each individual. The general HS profile shows similarity to the one proposed by Köse [2]. To our knowledge, we are the first to present reports regarding the precision of a HS segmentation method using a waist worn IMU.

\section{Conclusions And Future Directions}

Identifying HS events is important for determining bilateral gait parameter. We have shown that using a waist-worn Inertial Measurement Unit (IMU) on the ML axis, gait parameters and gait events, especially LHS and RHS, can be estimated. We have proposed a simple, adaptive, and parameter-free method for HS segmentation. Our proposed method using ACF, Wavelet features and RCE yields promising results based on our experimental data. In the near future we plan to investigate variations of the method outlined in this paper. 
TABLE I: Experimental summary of the proposed method on each subject

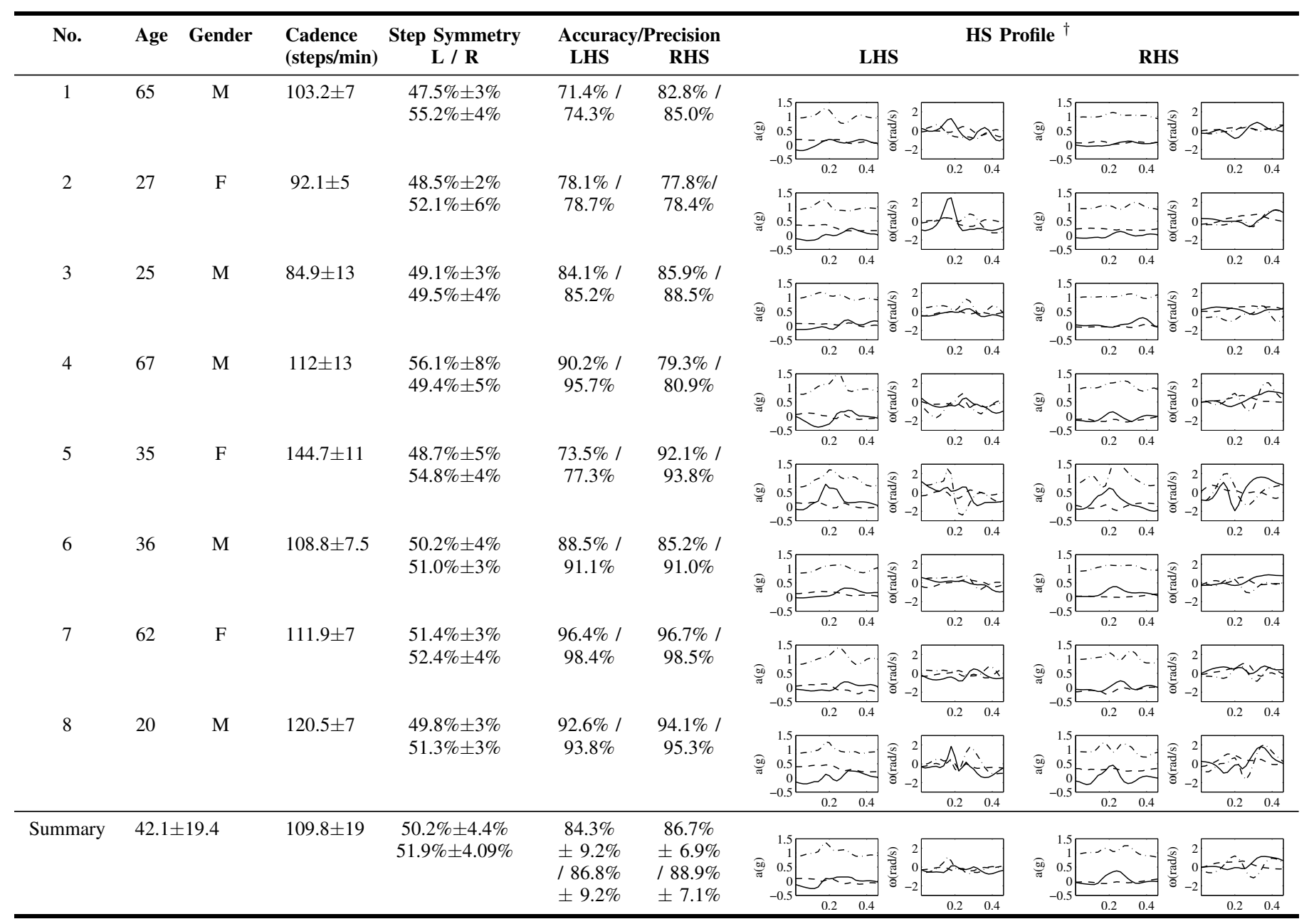

$\dagger \quad .-: a_{V} ; \omega_{T} \quad-: a_{A P} ; \omega_{C} \quad---: a_{M L} ; \omega_{S} \quad \mathrm{x}$ axis labels are $t(s)$

\section{REFERENCES}

[1] R. Moe-Nilssen and J. L. Helbostad, "Estimation of gait cycle characteristics by trunk accelerometry," Journal of Biomechanics, vol. 37, no. 1, pp. 121-126, 2004.

[2] A. Köse, A. Cereatti, and U. D. Croce, "Bilateral step length estimation using a single inertial measurement unit attached to the pelvis," Journal of NeuroEngineering and Rehabilitation, vol. 9, no. 9, 2012. [Online]. Available: http://www.jneuroengrehab.com/content/9/1/9

[3] A. Tura, M. Raggi, L. Rocchi, A. G. Cutti, and L. Chiari, "Gait symmetry and regularity in transfemoral amputees assessed by trunk accelerations," Journal of NeuroEngineering and Rehabilitation, vol. 7, no. 4, 2010. [Online]. Available: http://www.jneuroengrehab.com/content/7/1/4

[4] P. Forsman, E. Toppila, and E. Haeggstrom, "Wavelet analysis to detect gait events," in Proc. of the Annual International Conference of the IEEE EMBS, sept. 2009, pp. 424-427.

[5] M. Patterson and B. Caulfield, "Comparing adaptive algorithms to measure temporal gait parameters using lower body mounted inertial sensors," in Proc. of the Annual International Conference of the IEEE EMBS, Aug. 28-Sept. 1 2012, pp. 4509-4512.

[6] I. Pappas, T. Keller, S. Mangold, M. Popovic, V. Dietz, and M. Morari, "A reliable gyroscope-based gait-phase detection sensor embedded in a shoe insole," IEEE Sensors, vol. 4, no. 2, pp. 268-274, april 2004.

[7] M. D'Amico, R. Bellomo, R. Saggini, and P. Roncoletta, "A 3d spine \& full skeleton model for multi-sensor biomechanical analysis in posture \& gait," in Proc of the IEEE International Workshop on Medical Measurements and Applications (MeMeA), may 2011, pp. 605-608.
[8] F. Bugané, M. Benedetti, G. Casadio, S. Attala, F. Biagi, M. Manca, and A. Leardini, "Estimation of spatial-temporal gait parameters in level walking based on a single accelerometer: Validation on normal subjects by standard gait analysis," Computer Methods and Programs in Biomedicine, vol. 108, no. 1, pp. 129-137, 2012.

[9] S. Research. (2010) Kinematics guide revision 1e. [Online]. Available: http://www.shimmer-research.com/wp-content/uploads/2010/09/

[10] M. Henriksen, H. Lund, R. Moe-Nilssen, H. Bliddal, and B. DanneskiodSamse, "Test-retest reliability of trunk accelerometric gait analysis," Gait \& Posture, vol. 19, no. 3, pp. 288 - 297, 2004.

[11] M. Yuwono, S. W. Su, B. D. Moulton, and H. T. Nguyen, "Gait episode identification based on wavelet feature clustering of spectrogram images," in Proc. of the 34rd Annual International Conference of the IEEE EMBS, San Diego, Aug. 28-sept. 1 2012, pp. 2949-2952.

[12] N. Boulgouris, D. Hatzinakos, and K. Plataniotis, "Gait recognition: a challenging signal processing technology for biometric identification," IEEE Signal Processing Magazine, vol. 22, no. 6, pp. 78 - 90, nov. 2005.

[13] M. Yuwono, S. W. Su, B. D. Moulton, and H. T. Nguyen, "Fast unsupervised learning method for rapid estimation of cluster centroids," in Proc. of the 2012 IEEE Congress on Evolutionary Computation, June 10-15 2012, pp. 889-896.

[14] M. Yuwono, S. W. Su, B. D. Moulton, and H. T. Nguyen, "Optimization strategies for rapid centroid estimation," in Proc. of the 34rd Annual International Conference of the IEEE EMBS, San Diego, Aug. 28-sept. 12012 , pp. 6212-6215 